\title{
A CLINICAL STUDY ON ACUTE PANCREATITIS AND ITS DIFFERENT ETIOLOGIES IN BOWRING \& LADY CURZON HOSPITALS, BENGALURU
}

\author{
Harindranath H. R', Narendranath $L^{2}$
}

${ }^{1}$ Associate Professor, Department of General Surgery, Bangalore Medical College \& Research Institute.

${ }^{2}$ Post Graduate, Department of General Surgery, Bangalore Medical College \& Research Institute.

\begin{abstract}
There are various etiological factors causing acute pancreatitis; of them to identify the most common etiological factor affecting the severity of symptoms, complications and mortality in acute pancreatitis. Acute pancreatitis is one of the commonest medical emergencies encountered in Tertiary Care Center. Different etiologies have been proposed in its causation in different parts of the world, i.e. alcohol, gallstones, viruses, certain drugs; however, there are no much studies conducted in India to assess the incidence of acute pancreatitis and the role of different etiological agents.
\end{abstract}

\section{METHODS}

This study was conducted in Bowring and Lady Curzon hospital, Bengaluru. A total of 40 patients admitted between January 2015 to November 2015 with symptoms of acute pancreatitis were analysed retrospectively to study the etiology in each case and findings were tabulated.

\section{RESULTS AND CONCLUSION}

The highest incidence of acute pancreatitis was found in the age group of 30 to 50 years followed by the age group of 20 to 30 years. Acute pancreatitis was found more commonly in males compared to females. This may be due to effect of alcohol addiction in males. Surgeries not much useful except in cases of gallstone and necrotizing pancreatitis. In acute pancreatitis, serum lipase level may be elevated more consistently and for longer half-life than serum amylase. Octreotide used in all patients, found helpful in producing symptomatic relief and promoting recovery in mild to moderate cases.

\section{KEYWORDS}

Acute Pancreatitis, Alcohol, Gallstones, Serum Amylase, Serum Lipase.

HOW TO CITE THIS ARTICLE: Harindranath H. R, Narendranath L. A clinical study on acute pancreatitis and its different etiologies in bowring \& lady Curzon hospitals, Bengaluru. J Evolution Med Dent Sci 2016;5(3):196-199, DOI: 10.14260/jemds/2016/44

\section{INTRODUCTION}

Acute pancreatitis is a common cause of acute abdominal pain requiring hospital admission. The attack is mild in about $75 \%$ of patients, who will show marked improvement within $48-72$ hours. In some $20 \%$ of patients; however it is often severe with high morbidity and mortality.[1] The first 12 hours are extremely important to provide appropriate management, which will decrease morbidity and mortality. Nearly $80 \%$ of cases of Acute Pancreatitis worldwide are caused by gallstone obstruction and high alcohol intake. It is necessary to identify the etiology to institute definitive management and prevent further attacks. There appears to be an increase in the incidence of acute pancreatitis. This rise in incidence has been attributed to increased alcohol consumption and high calorie food consumption but may well reflect improved diagnostic capability during this period.[2] No seasonal or weekly pattern of acute pancreatitis has been observed. Men are affected much more than women. ${ }^{[3]}$

\section{BILIARY PANCREATITIS}

The high incidence of common bile duct stones (28\%) supports the hypothesis that the causative mechanism in gall stone pancreatitis is the passage of a calculus through the common channel formed by pancreatic and common bile ducts, with consequent reflux of bile or duodenal juice into the pancreatic duct.[4],[5]

Financial or Other, Competing Interest: None.

Submission 08-12-2015, Peer Review 09-12-2015,

Acceptance 18-12-2015, Published 09-01-2016.

Corresponding Author:

Dr. Harindranath H. $R$,

18/52, $1^{\text {ST }}$ Cross,

Pakash Nagar,

Bangalore-560021.

E-mail: harindranath82@gmail.com

DOI:10.14260/jemds/2015/44
Impaction of a stone at the ampula of Vater in $56 \%$ of necropsies showing gall stone pancreatitis lends credence to this theory and suggests that early operative intervention should be considered. Incidence is highest in patients with small gallstones or microlithiasis, as these stones are more likely to escape the gallbladder and transit the cystic duct to reach the common bile duct.[6] Large stones are more likely to be retained in the gallbladder. A recent study noted that the incidence of all causes of acute pancreatitis rose between 1994 and 2001 from 33.2 cases to 43.8 cases per 100,000 adults, without a reduction of mortality rate. This increase was attributed to a rise in the incidence of gallstone pancreatitis. ${ }^{[7]}$

\section{ALCOHOLIC PANCREATITIS}

Alcohol is a well-known precipitant of acute pancreatitis, although the incidence of acute pancreatitis in heavy alcohol consumers is not more than $2 \%$ to $3 \%$ per year, suggesting that there are as yet undetermined environmental or genetic factors that influence the development of acute pancreatitis in this population. ${ }^{[8]}$ The incidence rate may be similar in heavy drinkers of both sexes. Acute pancreatitis incidence rates peak between ages 35 and 44 years.

Furthermore, alcoholic acute pancreatitis has the highest associated risk of overall mortality, with the odds of death increased $90 \%$ as compared with biliary pancreatitis, possibly due to poor baseline nutrition. ${ }^{[9]}$ Excessive alcohol use as a cause of pancreatitis is more common among men than women; the association between alcohol consumption and acute pancreatitis is complex but appears to be dosedependent. An increase in acute pancreatitis secondary to chronic alcoholism was confirmed by a study conducted by Trapnel and Duncan.[10] Other causes of Acute pancreatitis are hyperlipidemia, hyperparathyroidism and ERCP.[11],[12] 
A retrospective cohort study conducted by Rebecca A. Noel showed an increased risk of acute pancreatitis and biliary disease observed in patients with Type 2 diabetes.[13] In 10\% of cases is idiopathic acute pancreatitis where no underlying cause can be identified.[5]

\section{METHODS AND METHODOLOGY}

This study was conducted in 40 patients of acute pancreatitis admitted in Bowring and Lady Curzon Hospital from 1st January to 1st December 2015 attached to Bangalore Medical College and Research Institute; 40 cases of acute pancreatitis were admitted, investigated and treated. Observations were made with regards to common presentation, sex distribution, common etiology and common blood parameters.

All patients were assessed, managed either conservatively, findings were recorded. Proforma was designed to record the history, chief complaint, past history, family history, personal history, diet history, alcohol consumption, physical examination, local examination, operative history, investigations and management. The clinical presentation associated medical conditions, laboratory and radiological investigations, severity, management and outcome were studied.

The attack was categorized as severe if three or more of the parameters were present during the first 48 hours of admission as described by Ranson et al.[14] Ten patients with Ranson's score $>3$ and those with serious co-morbidity were admitted to ICU and monitored. All patients had full blood count and blood chemistry including serum amylase estimation done and biliary pancreatitis was diagnosed when biliary stones were demonstrated by ultrasonography which was performed within 24 hours of admission. CT scan was performed on patients. All patients were treated conservatively.

\section{RESULTS AND DISCUSSION}

In the study of 40 cases of acute pancreatitis following observations are made. Age Group: Most commonly affected age group in this study is 20-49 years and mean age is 34 years. As compared to females, male patients are more affected by acute pancreatitis in my study. 10 patients (25\%) had one or more comorbidities like type 2 diabetes mellitus or hypertension. The mean Ranson's criteria were $2 \pm 1.08$. Ranson's score of 3 or more was found in 12 (30\%) patients and was 2 or less in $28(70 \%)$ patients. Abdominal ultrasonography revealed calculi in the gall bladder of 11 $(27.5 \%)$ patients; choledocholithiasis in $6(15 \%)$ patients; pancreatic enlargement in 32 (80\%); pancreatic pseudocyst in $4(10 \%)$ patients; dilated common bile duct $>7 \mathrm{~mm}$ in 5 $(12.5 \%)$ patients; and ascites in 15 (37.5\%). In order of occurrence, commonest causes in males is alcoholism 28 $(70 \%)$, followed by biliary diseases 11 (27.5\%). While in females biliary tract disease $(80 \%)$ leads. As in our society alcoholism is not that common in females.

\section{PRESENTING SYMPTOMS}

From the table below it is evident that abdominal pain is the commonest presenting symptom, present in almost all the patients, of these $70 \%$ of patients presented with typical epigastric pain that radiate to the back.[1] Physical Findings: In my study fever (25\%) and tachycardia (60\%) are most common vital derangement seen in patients with acute pancreatitis.[15] Epigastric tenderness is found in almost all patients. In $30 \%$ patients abdominal wall guarding and rigidity found. Surprisingly, pallor found in $40 \%$ of patients along with icterus in $5 \%$ of patients.

\begin{tabular}{|c|c|c|}
\hline & No. of Patients & Percentage \\
\hline Abdominal pain & 40 & 100 \\
\hline Abdominal distension & 8 & 20 \\
\hline Vomiting & 22 & 55 \\
\hline Fever & 10 & 25 \\
\hline Dyspnea & 5 & 12.5 \\
\hline Jaundice & 2 & 5 \\
\hline Shock & 2 & 5 \\
\hline
\end{tabular}

\section{ETIOLOGY}

\begin{tabular}{|c|c|c|}
\hline & No. of Patients & Percentage \\
\hline Alcohol & 28 & 70 \\
\hline Gall stones & 11 & 27.5 \\
\hline Both & 5 & 12.5 \\
\hline Others & 5 & 12.5 \\
\hline
\end{tabular}

Commonest causes are alcoholism and biliary tract disease accounting more than $90 \%$ of acute pancreatitis.[16][17] Idiopathic acute pancreatitis are third most common causes.

\section{BLOOD INVESTIGATIONS}

\section{Serum Amylase}

It is evident from this study that, the patient with systemic complication pre-dominance, hyperamylasemia is not that evident. While in those patients with local pancreatitis, serum amylase was significantly high at the time of diagnosis and remains high for 7-10 days at least. Increased level of serum amylase is essential feature of acute pancreatitis. But the pattern of the hyperamylasemia is extremely variable. Pattern increase or decrease, does not correspond with the severity, complications or treatment modality.

\begin{tabular}{|c|c|c|}
\hline S. Amylase & No. of Patients & Percentage \\
\hline$<80$ & 4 & 10 \\
\hline $80-499$ & 10 & 25 \\
\hline $500-999$ & 18 & 45 \\
\hline$>1000$ & 8 & 20 \\
\hline
\end{tabular}

Above table shows values of S. amylase level is elevated in most of the patients $(90 \%)$ during the initial period of Acute Pancreatitis. Thus hyperamylasemia is a highly sensitive but not specific marker of disease.[18]

\section{Serum lipase}

\begin{tabular}{|c|c|c|}
\hline S. Lipase & No. of Patients & Percentage \\
\hline$<80$ & 6 & 15 \\
\hline $80-499$ & 9 & 22.5 \\
\hline $500-999$ & 15 & 37.5 \\
\hline$>1000$ & 10 & 25 \\
\hline
\end{tabular}

Serum lipase is only secreted by the pancreas and thus more specific. In acute pancreatitis, serum lipase level may be elevated more consistently and for longer half-life than serum amylase. In my study it is seen elevated in $85 \%$ of patients.[19] Biochemical tests were done in this study. Elevated TLC was suggestive of the infection in the pancreas but it was nonspecific. Altered renal function test in about 6 patients was due to systemic complications of acute pancreatitis which lead to shock and renal failure. S. bilirubin, ALP, AST \& ALT were elevated in 10 cases of Acute pancreatitis due to biliary tract disease or due to pathology of the pancreas involving the head and peri-ampullary region. ${ }^{[20]}$ In majority of patients X-Ray abdomen did not reveal any information about the diagnosis. Raised hemidiaphragm may be seen due to either pancreatic or retro-pancreatic edema or due to pseudocyst. 
Pleural effusion was present as the systemic complications of acute pancreatitis in 6 patients.

\section{Ultra-Sonography}

USG was performed as and when required in all the patients of this study. Thus it is evident that almost all types of acute pancreatitis can be diagnosed by ultrasonography, as USG has high sensitivity.[21]

\begin{tabular}{|c|c|c|}
\hline USG Findings & $\begin{array}{c}\text { No. of } \\
\text { Patients }\end{array}$ & Percentage \\
\hline $\begin{array}{c}\text { Bulky pancreas with } \\
\text { peripancreatic fluid } \\
\text { collection }\end{array}$ & 32 & 80 \\
\hline Altered echotexture & 8 & 20 \\
\hline Fatty liver & 16 & 40 \\
\hline Gall bladder stones & 11 & 27.5 \\
\hline Ascites & 15 & 37.5 \\
\hline Pleural effusion & 6 & 15 \\
\hline
\end{tabular}

\section{CECT Abdomen}

Though contrast enhanced computerized tomography (CECT) abdomen is most valuable tool for diagnosis of acute pancreatitis and its local complication. Out of 40 patients, only 08 patients had undergone these investigations. Since it is costly rest of the patients could not afford it. The modified CT severity index scoring of these patients ranges from 1 to 10 Patients with high CT severity index had high morbidity and mortality. Patients with low CT severity index had no mortality.[22]

\section{MANAGEMENT}

The principles of conservative management followed in each case were adequate analgesia, correction of fluid and electrolytes imbalance, complete GI tract rest and Antibiotics.[23] Octreotide, somatostatin analog was used in all patients. In this study octreotide was found helpful in producing symptomatic relief and promoting recovery in mild to moderate Acute pancreatitis.[24] But not much helpful in severe cases. Ryle's tube was inserted in all patients was found to decrease vomiting, protect patients from aspiration and decompress the gut in ileus. Continuous radiological, biochemical and clinical monitoring was done in every case to prevent complications and for better prognosis of the patients.

\section{COMPLICATIONS}

\begin{tabular}{|c|c|c|}
\hline & $\begin{array}{c}\text { No. of } \\
\text { Patients }\end{array}$ & Percentage \\
\hline Infection/sepsis & 6 & 15 \\
\hline Respiratory /ARDS & 1 & 2.5 \\
\hline Renal/AKI & 2 & 5 \\
\hline Pseudocyst & 4 & 10 \\
\hline Peripancreatic fluid collection & 8 & 20 \\
\hline Pancreatic necrosis & 1 & 2.5 \\
\hline Death & 1 & 2.5 \\
\hline
\end{tabular}

During this study, total $57 \%$ of patients developed complications. Ascites- 15 patients, Pseudocyst - 4 patients, recurrent attacks-18 (45\%) patients.

\section{CONCLUSION}

The highest incidence of acute pancreatitis was found in the age group of 30 to 49 years followed by the age group of 20 to 49 years. It was relatively less common in the extremes of age groups. Acute pancreatitis was found more commonly in males compared to females. This may be due to effect of alcohol addiction in males. Alcoholism and biliary tract diseases are the commonest factors in etiology of acute pancreatitis. [25] Amongst which males were affected most by alcoholism and females by biliary tract disease is common.

Acute pancreatitis usually presents with abdominal pain, nausea, vomiting, fever, jaundice and abdominal distention and physical examination reveals epigastric tenderness, localised guarding, pyrexia and shock mostly.

Most attacks of acute pancreatitis were of the mild type. The most common systemic complications were pulmonary, followed by renal complications like ARF. DIC was found in one patient. The most common local complications were acute fluid collection and pseudo-pancreatic cyst. Pancreatic necrosis, pancreatic abscess were found only in patients having severe pancreatitis. Serum amylase and serum lipase are the best biochemical indicators of the disease, supplemented by serial ultrasound examinations of the abdomen. Computed tomography is not used routinely due to its high cost.

The prognostic factors for this disease are hyperamylasemia, hyperglycemia, reflecting a poorer outlook. Prognosis can also be assessed by ultrasonography and computed tomography delineating local complications. USG abdomen is a good, readily available, non-invasive mean to detect local pathology, which may be readily repeated. CECT abdomen is costly, invasive procedure, but it is very sensitive and specific for detection of local pathology. In the study, only 8 patients had undergone CT scan because the cost of investigation is very high. Peri-pancreatic fluid collection and pancreatic necrosis are predictors of severity of pancreatitis on USG and CT scan findings. Serum amylase level more than threefold of normal level and peri-pancreatic fluid collection is main predictor of severity of pancreatitis in my study.

About $90 \%$ of patients were treated conservatively. octreotide was used in all patients and was found effective in providing symptomatic relief and promoting recovery in patients with milder form of disease. The initial management of Acute Pancreatitis should be conservative with surgery reserved for trauma, very severe attack not responding to medical therapy and complications of disease.

\section{REFERENCES}

1. Ryan Van Woerkom, Douglas G Adler. Acute Pancreatitis: Review and Clinical Update. Hospital Physician. 2009;45(1):9-19.

2. Timothy B Gardner, Santhi Swaroop Vege, Suresh T Chari, Randall K Pearson, Jonathan E Clain, Mark D Topazian, et al. The effect of age on hospital outcomes in severe acute pancreatitis. Pancreatology 2008;8:265-270.

3. Baig SJ, Rahed A, Sen S. A prospective study of the etiology, severity and outcome of acute pancreatitis in Eastern India. Trop Gastroenterol. 2008;29(1):20-2.

4. Trapnell JE, Duncan EHL. Patterns of incidence in Acute pancreatitis. Br Med J 1975;2:179-83.

5. Acosta JM, Pellegrini CA, Skinner DB. Etiology and pathogenesis of acute biliary pancreatitis. Surgery 1980;88:118-25.

6. Saraswat VA, Sharma BC, Agarwal DK, Kumar R, Negi TS, Tandon RK. Biliary microlithiasis in patients with idiopathic acute pancreatitis (RIAP) and unexplained biliary pain: response to therapy. J Gastroenterol Hepatol. 2004;19(10):1206-11.

7. Juan Miguel Acosta and Carlos Luis Ledesma. Gallstone Migration as a Cause of Acute Pancreatitis. N Engl J Med 1974;290:484-487.

8. Frey CF, Zhou H, Harvey DJ, et al. The incidence and casefatality rates of acute biliary, alcoholic and idiopathic pancreatitis in California, 1994-2001. Pancreas 2006;33:336-44. 
9. Elta GH. Sphincter of Oddi dysfunction and bile duct microlithiasis in acute idiopathic pancreatitis. World J Gastroenterol 2008;14:1023-6. 22.

10. David C Whitcomb. Acute Pancreatitis. New England Journal of Medicine; 2006;20(354):2142-2150.

11. Steinberg WM, Lewis JH. Steroid-induced pancreatitis: does it really exist? Gastroenterology 1981;81:799-808.

12. Bourke JB. Incidence and mortality of acute pancreatitis. Br Med J 1977;2:1668-1669.

13. Ranson JHC. Acute pancreatitis - where are we? Surg Clin N Am 1981;61:55-70.

14. Sakorafas, George H Tsiotou, Adelais G. Clinical Reviews: pancreatic and biliary diseases. Etiology and Pathogenesis of Acute Pancreatitis: Current Concepts. J of Clinl Gast 2000;30(4):343-356.

15. Benifla, Mony, Weizman, et al. Liver, pancreas and biliary tract: clinical research. Acute Pancreatitis in Childhood: Analysis of Literature Data, J of Clin Gast 2003;37(2):169-172.

16. Corfield AP, Cooper MJ, Williamson RC. Acute pancreatitis: a lethal disease of increasing incidence. Gut 1985;26:724-729.

17. Bohidar NP, Pramod K Garg, Sudeep Khanna, et al. Incidence, etiology and impact of fever in patients with acute pancreatitis pancreatology. 2003;3:9-13.
18. Jaakkola M, Nordback I, Sillanaukee P, et al. Blood tests for detection of alcoholic cause of acute pancreatitis, Lancet 1994;343:1328-29.

19. Jennifer K, Carrol, Brian Herrick, et al. Acute pancreatitis: diagnosis, prognosis and treatment. AmFam Physician 2007;75:1513-20.

20. Andrew Kingsnorth, Derek O'Reilly. Acute pancreatitis. BMJ 2006;332:1072.

21. Catalano MF, Lahoti S, Geenen JE, et al. Prospective evaluation of endoscopic ultrasonography, endoscopic retrograde pancreatography, in the diagnosis of acute pancreatitis. Gastrointest Endosc. 1998;48:11-17.

22. Balthazar EJ, Robinson DL, Megibow AJ, et al. Acute pancreatitis: value of CT in establishing prognosis. Radiology, 1990;174:331-336.

23. Gardner TB, Vege SS, Pearson RK, et al. Fluid resuscitation in acute pancreatitis. Clin Gastroenterol Hepatol. 2008;6:1070-1076.

24. Toskes PP, Forsmark CE, DeMeo MT, et al. A multicenter controlled trial of octreotide for pain of chronic pancreatitis. Pancreas 8.1993; A-774.

25. Yeo CJ, Cameron JL: Exocrine pancreas, in Townsend CM et al. (eds): Sabiston's Textbook of Surgery. Philadelphia: Saunders, 2000, p 1117. 143. 